\title{
Less invasive lumbopelvic fixation technique using a percutaneous pedicle screw system for unstable pelvic ring fracture in a patient with severe multiple traumas
}

\author{
Sei Yano, MD, ${ }^{1}$ Yasuchika Aoki, MD, ${ }^{1,2}$ Atsuya Watanabe, MD, ${ }^{1,2}$ Takayuki Nakajima, MD, ${ }^{1,2}$ \\ Makoto Takazawa, MD, ${ }^{1,2}$ Hiroyuki Hirasawa, MD, ${ }^{3}$ Kazuhisa Takahashi, MD, ${ }^{4}$ \\ Koichi Nakagawa, MD, ${ }^{5}$ Arata Nakajima, MD, ${ }^{5}$ Hiroshi Takahashi, MD, ${ }^{5}$ Sumihisa Orita, MD, ${ }^{4}$ \\ Yawara Eguchi, MD, ${ }^{4}$ Takane Suzuki, MD, ${ }^{4}$ and Seiji Ohtori, MD ${ }^{4}$
}

\begin{abstract}
Departments of ${ }^{1}$ Orthopaedic Surgery and ${ }^{3}$ Emergency and Critical Care Medicine, Eastern Chiba Medical Center, Togane; Departments of ${ }^{2}$ General Medical Science and ${ }^{4}$ Orthopaedic Surgery, Graduate School of Medicine, Chiba University, Chiba-city; and ${ }^{5}$ Department of Orthopaedic Surgery, Toho University Sakura Medical Center, Sakura, Chiba, Japan
\end{abstract}

\begin{abstract}
Pelvic ring fractures are defined as life-threatening injuries that can be treated surgically with external or internal fixation. The authors report on an 81-year-old woman with an unstable pelvic fracture accompanying multiple traumas that was successfully treated with a less invasive procedure. The patient was injured in a traffic accident and sustained a total of 20 fractures, including pelvic ring, bilateral rib, and lumbar transverse processes fractures, and multiple fractures of both upper and lower extremities. The pelvic ring fracture was unstable with fractures of the bilateral sacrum with right sacroiliac disruption, right superior and inferior pubic rami, left superior pubic ramus, and ischium. During emergency surgery, bilateral external fixation was applied to the iliac crest to stabilize the pelvic ring. Second and third surgeries were performed 11 and 18 days after the first emergency surgery, respectively, to treat the multiple fractures. At the third surgery, the pelvic ring fracture was stabilized surgically using a less invasive posterior fixation technique. In this technique, 2 iliac screws were inserted on each side following an 8-cm midline posterior incision from the S-1 to S-3 spinous process, with the subcutaneous tissue detached from the fascia of the paraspinal muscles. The S-2 spinous process was removed and 2 rods were connected to bilateral iliac screws to stabilize the bilateral ilium in a switchback fashion. A crosslink device was applied to connect the 2 rods at the base of the S-2 spinous process. Following pelvic fixation, percutaneous pedicle screws were inserted into L-4 and L-5 vertebral bodies on both sides, and connected to the cranial rod connecting the bilateral iliac screws, thus completing the lumbopelvic fixation. The postoperative course was favorable with no postoperative complications. At the 10-month follow-up, bone union had been achieved at the superior ramus of the pubis, the patient did not complain of pain, and her activities of daily life returned to preinjury status. Unstable pelvic ring fractures need to be sufficiently stabilized for good surgical outcome. However, to avoid postoperative complications, a less invasive treatment is preferred, particularly in cases with poor general condition. This procedure is less invasive and provides sufficient stabilization to the unstable pelvic ring fracture, and thus is the ideal surgical procedure for such cases.
\end{abstract}

https://thejns.org/doi/abs/10.3171/2016.7.SPINE16323

KEY WORDS pelvic ring fracture; iliac screw; percutaneous pedicle screw; lumbopelvic; posterior fusion; switchback; surgical technique

$\mathrm{P}$ ELVIC ring fractures result from high-energy trauma and, because they may lead to massive bleeding resulting in hypovolemic shock, are considered to be the most serious fracture in terms of mortality rate..$^{2,8}$ Severe, unstable fractures are usually treated surgically with external or internal fixation. External fixation is used to temporarily stabilize unstable fractures and may reduce bleeding. Although bone healing can be obtained using external fixation in certain types of pelvic fracture, ${ }^{12}$ better outcomes are expected from internal fixation in severe cases with rotationally or vertically unstable fractures. ${ }^{9}$ Papakostidis et al. performed a systematic review and concluded that posterior pelvic internal fixation may yield better clinical results than nonoperative treatment

ABBREVIATIONS TAE $=$ transcatheter arterial embolization.

SUBMITTED April 16, 2016. ACCEPTED July 15, 2016.

INCLUDE WHEN CITING Published online October 7, 2016; DOI: 10.3171/2016.7.SPINE16323. 
and stabilization of the anterior pelvis. ${ }^{9}$ However, surgical exposure to perform posterior fixation produces a high wound complication rate. ${ }^{3,4,6,10}$ In particular, severe wound complications may occur when patients are treated with transcatheter arterial embolization (TAE) ${ }^{5,13}$ In this case report, we present a patient who suffered multiple traumas and an unstable pelvic ring fracture that was successfully treated using a less invasive posterior lumbopelvic fixation technique. Our procedure is described in detail, and the advantages of this technique compared with other procedures are discussed.

\section{Case Report}

\section{History, Examination, and Initial Treatment}

An 81-year-old woman was brought to the emergency room with multiple injuries from being hit by a motor vehicle while she rode her bicycle. On arrival, the patient was alert and oriented, but hemodynamically unstable. Intravenous solutions were rapidly administered until blood transfusions could be started. The initial radiographic examination showed a rotationally unstable pelvic ring fracture (Fig. 1A), and a CT scan revealed multiple fractures. A total of 19 fractures were found in addition to the pelvic ring fracture, including fractures of bilateral ribs and transverse processes of the lumbar spine, right clavicle, humerus (distal end) and radius (distal end), left clavicle, humeral head (with shoulder dislocation) and radius (distal end), right femoral greater trochanter, and left femoral trochanter and femoral shaft (compound fracture).

The lumbopelvic fractures were evaluated by multiplanar reconstruction $\mathrm{CT}$ including 3D reconstruction (Fig. 1B). We found fractures of the right L-3, L-4, and L-5 lumbar transverse processes, the bilateral sacrum with right sacroiliac disruption, the right superior and inferior pubic rami, and the left superior pubic ramus and ischium. After the CT scan, the patient was immediately transferred to the angiography suite for TAE. Following TAE, her hemodynamic condition became relatively stable and she was transferred to surgery.

\section{Emergency Surgery and Second and Third Surgeries}

Emergency surgery was started 4.5 hours after the time of injury. While the patient was under general anesthesia and intubated, bilateral external fixation was applied to the iliac crests to stabilize the pelvic ring (Fig. 1C). Second and third surgeries were performed 11 and 18 days, respectively, after the emergency surgery to stabilize the other fractures requiring surgical treatment. Written informed consent was obtained prior to each surgery.

At the third surgery, the pelvic ring fracture was stabilized surgically using our less invasive posterior fixation technique. The time for the third surgery was 223 minutes, the result of the measurement of intraoperative blood loss was $30 \mathrm{~g}$ during lumbopelvic fixation, and no intraoperative complications occurred.

\section{Third Surgical Technique}

The patient underwent surgery in the prone position after removal of external fixation. An 8-cm midline posterior incision from the S-1 to $\mathrm{S}-3$ spinous process exposed the
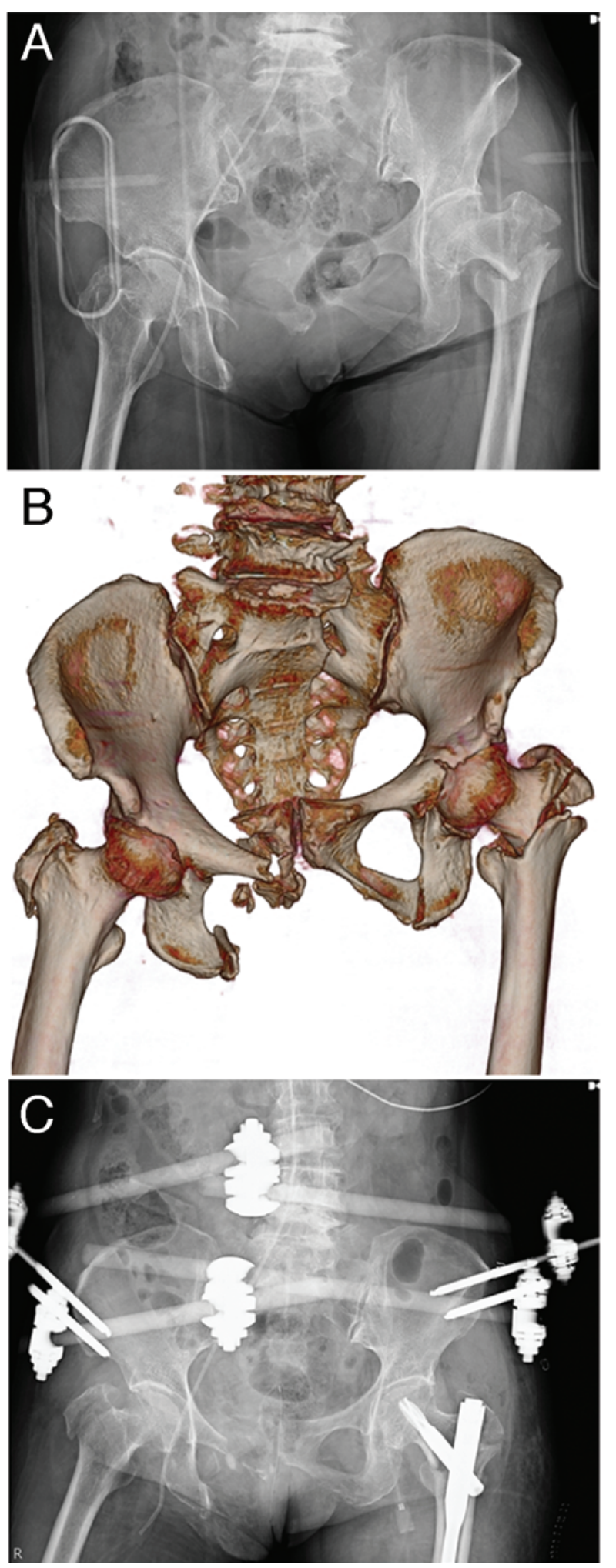

FIG. 1. A: Initial anteroposterior radiograph of the patient's pelvis showing the pelvic ring fracture with right sacroiliac dissociation and right pubic ramus fracture. B: Preoperative 3D CT reconstructed image of the pelvic ring showing right sacroiliac dissociation, right pubic ramus fracture, bilateral sacral fractures, and left superior pubic ramus and ischium fractures. C: Anteroposterior radiograph after the first surgery. The unstable pelvic ring fracture was stabilized by external fixation. Figure is available in color online only. 

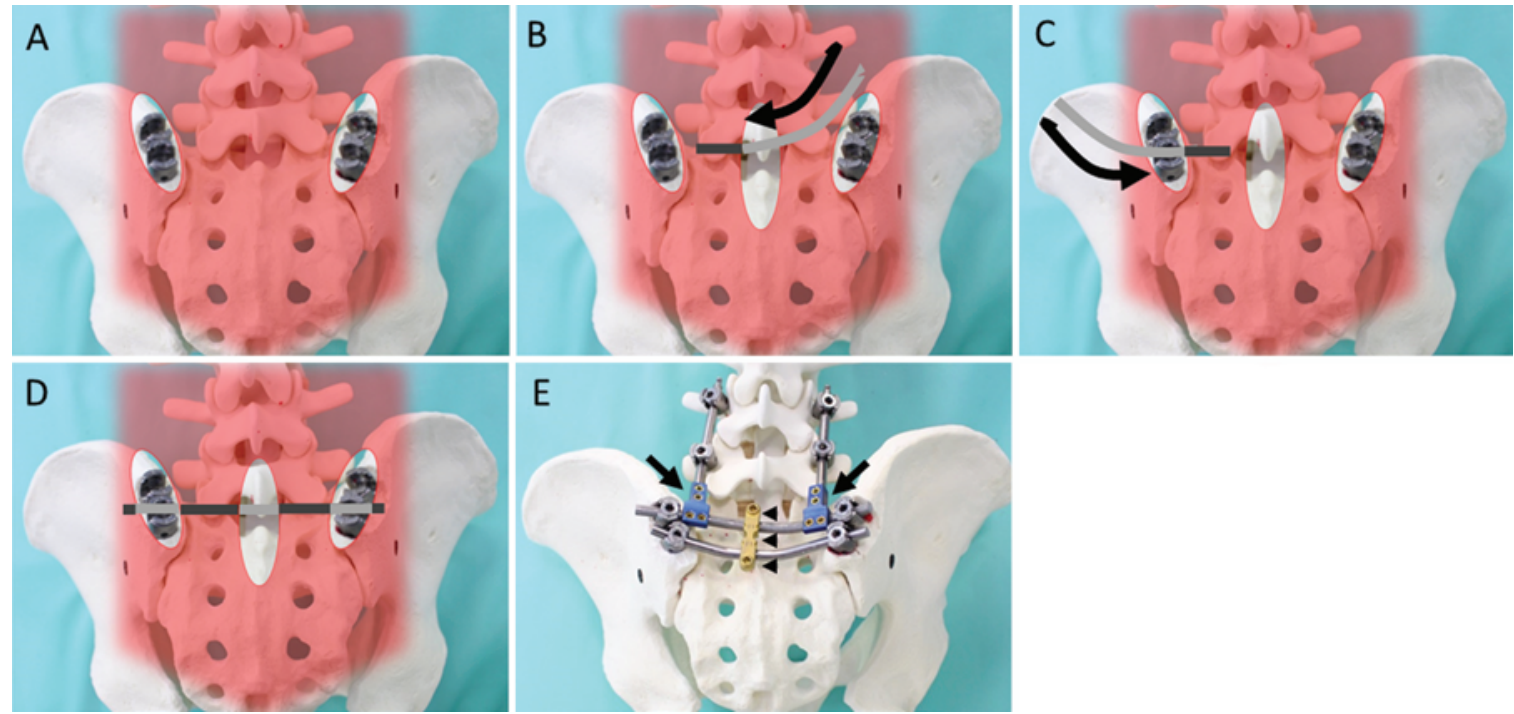

FIG. 2. Surgical techniques used for less invasive lumbopelvic fixation using a pelvic model. A: Two iliac screws are inserted on both sides from the midline incision. B: A rod is inserted (arrow) from the midline incision and passed under the left paraspinal muscles to the left iliac screw head. C: The rod is pushed further until the other end of the rod is located at the base of the S-2 spinous process. D: The rod is switched back and passed under the right paraspinal muscles to reach the right iliac screw head. E: Pedicle screws are inserted percutaneously to bilateral L- 4 and L-5 vertebrae, and rods are also inserted percutaneously to connect the pedicle screws to the iliac screw construct. The rods are connected to the iliac screw construct using a T-shaped rod connector (arrows). A crosslink device was applied (arrowheads) to stabilize the iliac screw construct, thus completing the lumbopelvic fixation. Figure is available in color online only.

fascia of the paraspinal muscles. Subcutaneous tissue was detached from the surface of the fascia and it was retracted to expose the bilateral posterior iliac crests. Fascia over the left posterior iliac crest was cut for a length of $3 \mathrm{~cm}$ to expose the insertion points of the iliac screws. Insertion points were determined using radiographic image guidance with the obturator outlet view showing a "teardrop target." 16 After partial removal of the posterior iliac wing to make a pit for the screw head, an intraosseous pathway was tunneled using a common pedicle screw probe, and 2 iliac screws were inserted (Expedium, DePuy Synthes Spine). Following left iliac screw placement, 2 iliac screws were inserted on the right side the same way as the left (Fig. 2A). Then, a 3-cm longitudinal incision of fascia was made to expose the S-2 spinous process. The S-2 spinous process was removed using a Liston bone cutter and the base of the process was flattened using a high-speed electric surgical drill. The distance between left and right iliac screws was measured with a rod template, and 2 rods were prepared to connect the left and right iliac screws. The rods were connected to bilateral iliac screws to stabilize the bilateral ilia by switchback placement (Fig. 2). One rod was inserted from the midline incision and passed under the left paraspinal muscles to the left iliac screw head (Fig. 2B). This rod was pushed further until the other end of the rod was located at the base of the S-2 spinous process (Fig. $2 \mathrm{C}$ ). Then the rod was switched back and passed under the right paraspinal muscles to reach the right iliac screw head (Fig. 2D). Another rod was inserted in the same way, and the 2 rods were connected to the corresponding iliac screw. A crosslink device was applied to connect the 2 rods at the base of the S-2 spinous process. Before tightening the rods to the iliac screws, displacement of the pelvic fracture was gently reduced by manually pressing the bilateral ilia. Following pelvic fixation, pedicle screws were inserted into L-4 and L-5 vertebral bodies on both sides. A 2-cm skin incision allowed insertion of pedicle screws percutaneously, using a percutaneous pedicle screw system (X-tab, DePuy Synthes Spine). A rod was inserted from the rod guide sleeve connected to the L-4 pedicle screw and advanced caudally through the L-5 rod guidance sleeve to the cranial rod connecting the bilateral iliac screws. The crossing point of the 2 rods was exposed by a $1-\mathrm{cm}$-long division of the paraspinal muscle, and the rods were connected using a T-shaped rod connector, completing the lumbopelvic fixation (Fig. 2E). Fascia and subcutaneous tissue were sutured, and the skin incision was closed using a skin stapler.

\section{Postoperative Course}

The postoperative course was favorable with no postoperative complications. A day after the third surgery, the patient could sit beside the bed and lie on the bed in a lateral decubitus position. One week after the third surgery, she could use a wheelchair. Because of the plate fixation treatment of the left femoral shaft fracture, no weight bearing was permitted on her left leg, except for toe-touch weight bearing, until 8 weeks after the third surgery. Twelve weeks after the third surgery, she was able to walk by herself using a walker and had only minor discomfort in the gluteal region.

At the 10-month follow-up examination, the patient reported no pain, and could walk with a walker. Her activities of daily life had returned to the status before the traffic accident. Radiographic examination revealed that bone union was achieved at the right superior ramus of 

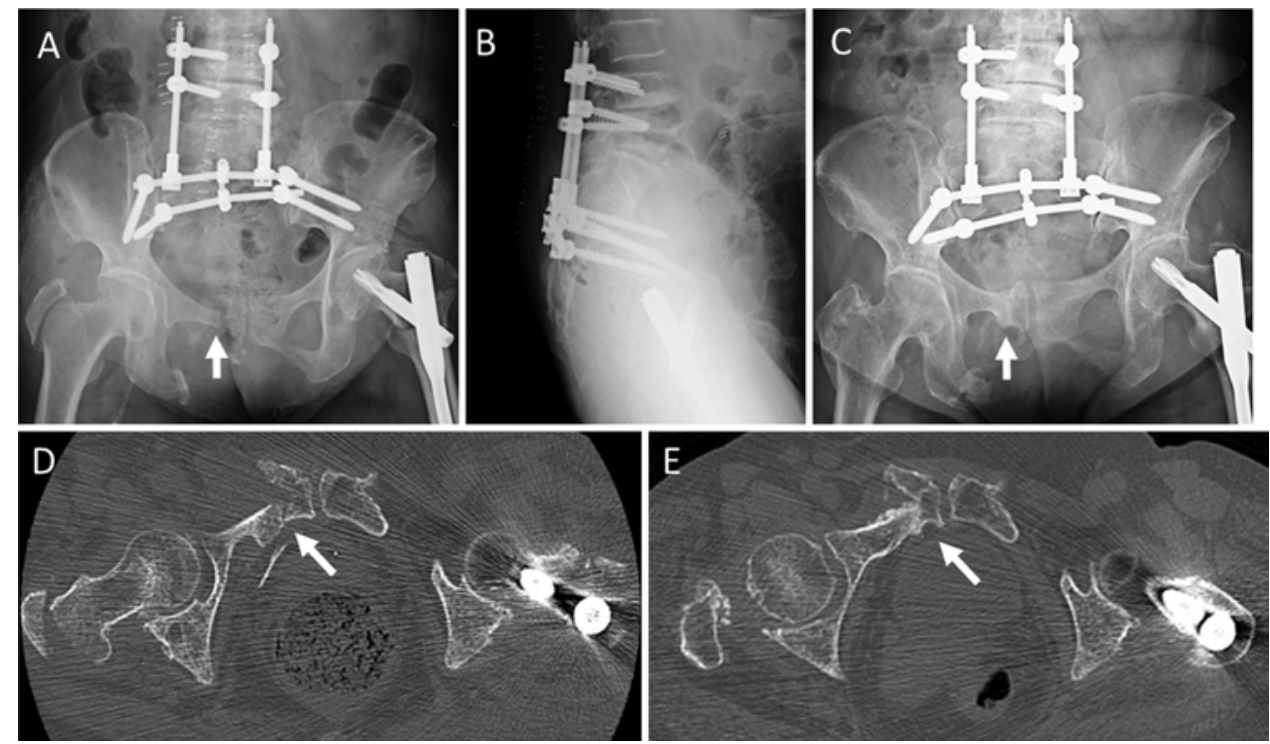

FIG. 3. Anteroposterior (A) and lateral (B) postoperative radiographs obtained on the day of the third surgery demonstrate the repositioned displaced pubic rami (arrow), compared with radiographs taken after the first surgery. An anteroposterior radiograph taken 10 months after the third surgery $(\mathbf{C})$ shows that bone union was achieved at the right superior pubic ramus (arrow). Axial postoperative CT images obtained at the third surgery (D) and 10 months after the third surgery (E); fracture healing at the right superior pubic ramus (arrows) is demonstrated at 10 months after the third surgery.

the pubis, but not the inferior ramus (Fig. 3). Because the patient had been treated by TAE, we were concerned that there might be poor blood supply to the muscle and skin. However, our less invasive surgery minimized iatrogenic tissue injury, and the surgical wound healed uneventfully without infection, necrosis, or other complications.

\section{Discussion}

Severe unstable pelvic fracture is usually treated surgically. For this case, 3 types of internal fixation methods were considered to stabilize this osteoligamentous injury: anterior plate placement using an ilioinguinal approach, sacroiliac screw fixation, and posterior lumbosacroiliac fixation using pedicle screw systems. ${ }^{14}$

A previously mentioned report suggested that posterior fixation produced better clinical results than stabilization of the anterior pelvis, despite the fact that posterior fixation tends to be applied to more severely unstable fractures. ${ }^{9}$ There are several posterior surgical procedures, and each procedure has merits and drawbacks. Sacroiliac screw fixation is simple, cheap, and less invasive. However, the results of a previous biomechanical study, using an unstable pelvic ring injury model with unilateral sacral fracture and pubic symphysis, suggest that the construct with bilateral single iliac screws connected by a rod was stiffer than single sacroiliac screw fixation, although it showed less stiffness than double sacroiliac screw fixation. ${ }^{15}$ Lumbopelvic fixation offers better stability, but has the disadvantages of surgical invasiveness and a higher complication rate. Common complications of lumbopelvic fixation are infection, wound-healing disturbances, and hardware issues, such as screw prominence and rod breakage. ${ }^{11}$ The risk of these complications appears to be higher when using lumbopelvic fixation than sacroiliac screw fixation. Moreover, our case was treated with TAE, thus these complications are more likely. ${ }^{5,13}$ Fortunately, our case had no postoperative complications because the patient was treated gently using a less invasive procedure. In addition, our case had bilateral sacral fractures, which occur secondary to a high-energy trauma, usually producing lumbopelvic instability. Thus, in our case, lumbopelvic fixation was desirable because the treatment goal for such cases is reconstruction of the lumbopelvic junction.?

The less invasive procedure using a percutaneous pedicle screw system has several advantages over conventional open procedures. Particularly in cases of multiple traumas, surgeons must make an effort to reduce surgical invasiveness to avoid worsening the general condition of patients. Koshimune et al. compared clinical results between their less invasive lumbopelvic fixation technique using a percutaneous pedicle screw system and the traditional open procedure. ${ }^{7}$ They inserted 2 iliac screws on each side connected to lumbar pedicle screws, and accomplished good lumbopelvic stabilization. Their technique reduced surgical time, intraoperative blood loss, and infection rates, indicating the usefulness of their less invasive procedure. However, the less invasive procedure using the percutaneous pedicle screw system has the disadvantage that the transverse connector could not be applied easily, as in the traditional open procedures. In cases with pelvic ring fracture with sacroiliac disruption, the aim of surgery is to connect the bilateral ilia to stabilize the disrupted pelvic ring. Without a transverse connector, bilateral ilia are connected indirectly using pedicle screws inserted into bilateral pedicles of the lumbar vertebrae. Better stability would be expected when bilateral iliac screws are directly connected by rods or transverse connectors. Dienstknecht et al. reported a minimally invasive stabilizing system for pelvic ring fracture. ${ }^{1}$ These authors applied a single iliac screw on each side and connected the screws by a rod in- 
serted subfascially with favorable clinical results. However, in cases with complex pelvic injuries, 14 of 16 patients had residual pain, and 12 of 16 cases experienced major restriction in their social or business life. We suggest that their procedure did not provide the sufficient stability found with our procedure. In our procedure, removal of the $\mathrm{S}-2$ spinous process provides several advantages. The transverse rods are easily inserted and connected to the bilateral iliac screws that are located deeper under the paraspinal muscles, reducing additional intraoperative softtissue damage and postoperative skin irritation. Although our procedure used a single $8-\mathrm{cm}$ midline skin incision, it could be performed using triple $3-\mathrm{cm}$ incisions, 1 for $\mathrm{S}-2$ spinous process removal and 2 for iliac screw placement. Most importantly, our procedure minimizes the soft-tissue damage that is closely related to postoperative complications. Moreover, our procedure provides good stability between the disrupted bilateral ilia. Yu et al. examined the stability of a spinopelvic construct using a total sacrectomy cadaver model; they reported that the construct with dual iliac screws showed much higher stability than that with a single iliac screw. ${ }^{17}$ In our procedure, 2 transverse rods can be easily inserted and connected by a crosslink device in the space created by removal of the S-2 spinous process, thus achieving good stability.

Severe unstable pelvic ring fracture must be sufficiently stabilized, however a less invasive treatment is preferred, particularly in cases with poor general conditions. Our procedure is one surgical option for treatment of unstable pelvic ring fracture that provides sufficient stabilization with less invasiveness.

\section{Acknowledgments}

We wish to thank Mr. Ryutaro Sakai and Ms. Tomomi Saito for help with the preparation of figures.

\section{References}

1. Dienstknecht T, Berner A, Lenich A, Nerlich M, Fuechtmeier B: A minimally invasive stabilizing system for dorsal pelvic ring injuries. Clin Orthop Relat Res 469:3209-3217, 2011

2. Gilliland MD, Ward RE, Barton RM, Miller PW, Duke JH: Factors affecting mortality in pelvic fractures. J Trauma 22:691-693, 1982

3. Hao T, Changwei Y, Qiulin Z: Treatment of posterior pelvic ring injuries with minimally invasive percutaneous plate osteosynthesis. Int Orthop 33:1435-1439, 2009

4. Jones CB, Sietsema DL, Hoffmann MF: Can lumbopelvic fixation salvage unstable complex sacral fractures? Clin Orthop Relat Res 470:2132-2141, 2012

5. Karadimas EJ, Nicolson T, Kakagia DD, Matthews SJ, Richards PJ, Giannoudis PV: Angiographic embolisation of pelvic ring injuries. Treatment algorithm and review of the literature. Int Orthop 35:1381-1390, 2011

6. Kellam JF, McMurtry RY, Paley D, Tile M: The unstable pelvic fracture. Operative treatment. Orthop Clin North Am 18:25-41, 1987

7. Koshimune K, Ito Y, Sugimoto Y, Kikuchi T, Morita T, Mizuno S, et al: Minimally invasive spino-pelvic fixation for unstable bilateral sacral fractures. Clin Spine Surg 29:124127,2016
8. O’Sullivan REM, White TO, Keating JF: Major pelvic fractures: identification of patients at high risk. J Bone Joint Surg Br 87:530-533, 2005

9. Papakostidis C, Kanakaris NK, Kontakis G, Giannoudis PV: Pelvic ring disruptions: treatment modalities and analysis of outcomes. Int Orthop 33:329-338, 2009

10. Routt ML Jr, Simonian PT, Mills WJ: Iliosacral screw fixation: early complications of the percutaneous technique. J Orthop Trauma 11:584-589, 1997

11. Soultanis K, Karaliotas GI, Mastrokalos D, Sakellariou VI, Starantzis KA, Soucacos PN: Lumbopelvic fracture-dislocation combined with unstable pelvic ring injury: one stage stabilisation with spinal instrumentation. Injury 42:1179-1183, 2011

12. Stahel PF, Mauffrey C, Smith WR, McKean J, Hao J, Burlew $\mathrm{CC}$, et al: External fixation for acute pelvic ring injuries: decision making and technical options. J Trauma Acute Care Surg 75:882-887, 2013

13. Suzuki T, Kataoka Y, Minehara H, Nakamura K, Uchino M, Kawai K, et al: Transcatheter arterial embolization for pelvic fractures may potentially cause a triad of sequela: gluteal necrosis, rectal necrosis, and lower limb paresis. J Trauma 65:1547-1550, 2008

14. Tonetti J: Management of recent unstable fractures of the pelvic ring. Orthop Traumatol Surg Res 99 (1 Suppl):S77_ S86, 2013

15. Vigdorchik JM, Jin X, Sethi A, Herzog DT, Oliphant BW, Yang KH, et al: A biomechanical study of standard posterior pelvic ring fixation versus a posterior pedicle screw construct. Injury 46:1491-1496, 2015

16. Wang MY: Percutaneous iliac screws for minimally invasive spinal deformity surgery. Minim Invasive Surg 2012:173685, 2012

17. Yu BS, Zhuang XM, Zheng ZM, Li ZM, Wang TP, Lu WW: Biomechanical advantages of dual over single iliac screws in lumbo-iliac fixation construct. Eur Spine J 19:1121-1128, 2010

\section{Disclosures}

The authors report no conflict of interest concerning the materials or methods used in this study or the findings specified in this paper.

\section{Author Contributions}

Conception and design: Aoki, Watanabe, T Nakajima, Takazawa, A Nakajima, Orita, Eguchi, Suzuki. Acquisition of data: Yano, T Nakajima, Takazawa, A Nakajima, H Takahashi, Orita. Drafting the article: Aoki, Yano. Reviewed submitted version of manuscript: all authors. Study supervision: Aoki, Hirasawa, K Takahashi, Nakagawa, Ohtori.

\section{Supplemental Information}

\section{Previous Presentations}

Portions of this work were presented in abstract form as proceedings at the 23rd Annual Meeting of the Japanese Society of Lumbar Spine Disorders, Tokyo, Japan, November 14-15, 2015.

\section{Correspondence}

Yasuchika Aoki, Department of Orthopaedic Surgery, Eastern Chiba Medical Center, 3-6-2 Okayamadai, Togane, Chiba 2838686, Japan.email: yasuaoki35@fc4.so-net.ne.jp. 